\title{
Structuring Goals and Measures for Information Management
}

\author{
André Minkus, Andreas Nobs and Sören Günther \\ ETH Zurich, Center for Enterprise Sciences (BWI) \\ Kreuzplatz 5, 8032 Zurich, Switzerland \\ aminkus@ethz.ch, anobs@ethz.ch, sguenther@ethz.ch \\ WWW home page: http://www.lim.ethz.ch
}

\begin{abstract}
Handling the complexity of Information Management (IM) is a very challenging issue - especially in global organizations. Such organizations consist of several entities with different strengths and weaknesses. They have to decide on common goals and agree on coordinated measures for improving their IM activities. However, many organizations lack the abilities to define and operationalize relevant goals. In addition, the complexity of existing measures makes it difficult for them to decide on those measures that are best suited for meeting their IM goals. This paper presents a methodology to structure goals and measures for improving IM. It starts on a strategic level and operationalizes both, goals and measures down to an application level. By considering human, organizational and technical aspects all relevant factors are included and interdependencies are highlighted. Organizations can benefit from the methodology since it supports the design of their IM activities and takes their specific strategies into consideration
\end{abstract}

Keywords

Information Management, Axiomatic Design Framework

\section{Introduction}

In recent years the importance of information management (IM) has increased significantly for many companies. IM refers to all activities connected to the acquisition, processing, transfer and provision of information [1]. It is seen as one of the most critical factors for business success. The growing number and complexity of the offered products and services have increased the amount of information to be exchanged. Up to now many companies solely rely on long-established forms of informal communication. This informal communication has proven to be very 
effective in local organizations. However, a growing globalization requires for more balanced channels of information exchange (IE). In many organizations different branches situated in different countries with different languages, cultures or time zones have to communicate with each other.

\subsection{Practitioners Problems and Requirements}

Typical examples for organizations like this are global service organizations of equipment manufacturing companies. They have a fast growing international customer base requiring them to establish new subsidiaries in new markets. These subsidiaries have to be supported with all information necessary for offering complex service products. In addition, they have to provide the companies headquarter with information about the performance of the products or about customer requirements.

In global organizations as presented above, information has to be exchanged on a just-in-time basis: at the right time, in a suitable format, at the right place, in the right quality and to the right person. For achieving this, organizations have to operationalize their IM goals and they have to define suitable measures. This requires to coordinate the different elements of the service organization (e.g. subsidiaries, partners) and to consider potential interactions between the different goals and measures. For many companies this imposes a major challenge.

For instance, in one company analyzed by the authors a lack of coordination has led to additional expenses and an increase of time necessary for exchanging information. In this organization, one major subsidiary had identified the need for supporting its IE by information and communication technology (ICT). An ICT solution was developed taking all needs of this subsidiary into consideration. In the same time, a similar solution was developed at one production location. Since these two solutions were developed independently they could only be used locally. Important information had to be transferred manually between them.

Another example illustrates the effects resulting from a lack of considering relevant interactions between different goals and measures. A central service division of a company decided to implement a system supporting different service technicians in their work at the customers' site. This ICT offered a variety of new functionalities. However, the characteristics of the process to be supported were not considered. For instance, using the solution required to enter a lot of data (e.g. equipment numbers) which was not always available at the customers' site. In addition, searching for information was complex and time consuming. This prevented a use in front of a waiting customer. Consequently, the technicians are still calling the hotline if they need any information. The ICT solution did not pay off.

\subsection{Overview of Relevant Literature}

A review on research literature shows a variety of existing concepts. For instance, there is literature focusing on strategies and goals of IM (e.g.[2]), addressing aspects of process design in information acquisition and provision (e.g.[3]) or presenting methods for supporting information exchange activities (e.g.[4]). While all these 
types of literature are crucial for the design of IM activities, there are some aspects missing. There exists no concept which structures IM objectives. Usually, the existing literature focuses either on strategic or operative levels but misses to link them. In addition, an analysis of interdependencies between different technical, organizational and human measures is missing. A description of those dependencies could reduce difficulties in selecting suitable measures by identifying necessary requirements and possible impacts of an implementation.

\subsection{Objective of the Paper}

Motivated by both, the practitioners requirements and the illustrated gap in literature this paper focuses on the development of an Information Management Factor Catalog (IMFC). This catalog structures the goals and measures relevant for improving IM in industrial service organizations and analysis their interactions. It aims to support companies in planning and setting up new structures, processes and methods for IM. Moreover, it can be used as a tool for identifying current weaknesses and potential for improvement.

\section{Methodology Used}

The research methodology used for the development of the IMFC bases on the principals of action research [5]. It involves the collaboration with four equipment manufacturing companies and their service organizations. For the design of the IMFC elements of the Axiomatic Design Framework (ADF) are used [6]. ADF was developed as a method for designing products and systems. As a fundamental characteristic ADF differentiates between goals ("what we want to achieve") and measures ("how we want to achieve it") [6]. To arrange goals and measures, it provides an illustration in form of a hierarchical tree. Two design axioms provide basic principles for generating a good design. Firstly, the independence axiom is stating that the independence of the different goals must be maintained. Secondly, the information axiom requires that the information content must be minimized and hence the design should be as simple as possible.

Using the ADF to develop the IMFC requires adapting one of its principles. Complete independence as stated in the first axiom can not be achieved because of the complex interdependencies between different organizational, technical and human aspects. Instead, the IMFC tries to reduce the dependencies. By arranging different goals and measures in a certain sequence, their dependencies can be reduced and handled.

\section{Setup of the IMFC}

The IMFC is structured in a tree diagram consisting of six levels [Fig. 1]. This chapter provides an overview on the proceeding when structuring goals and 
measures into the six levels. Since a complete explanation of the IMFC is too voluminous, this paper will focus on showing some examples.

\subsection{1st Level: Offering Competitive Products and Services as the Major Goal}

The first level refers to the most strategic goal influenced by IM activities. This goal is in line with the overall objectives of companies. It focuses on supporting the organization to offer superior products and services or to create a cost advantage [7]. One suitable measure helping to fulfill this goal is to provide the organization with all needed information at the right time, to the right person, in the right quality and a suitable format.

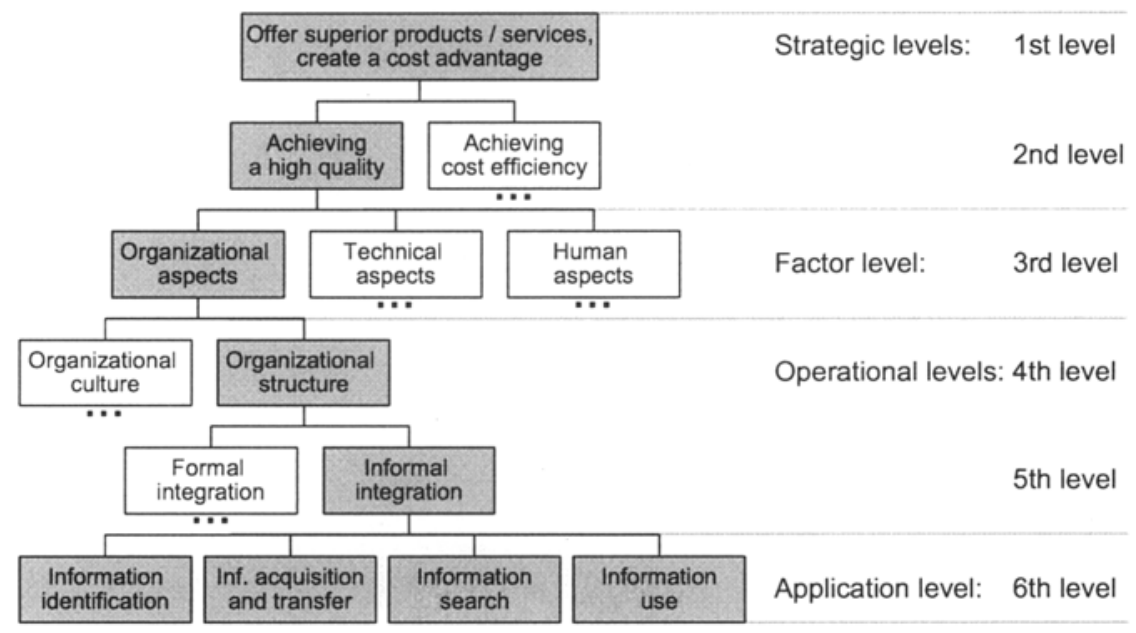

Fig. 1. Example showing the decomposition of the six levels of the IMC

\subsection{2nd Level: Achieving Quality and Cost Efficiency as Strategic Goals}

The first goal is decomposed in the second level by considering the different target areas of IM. Since exchanging information is a part of the logistics processes of an organization, the four target areas of logistics - quality, time, flexibility and costs [8] - can be used. However, in contrast to tangible products the quality of information includes the dimensions of time and flexibility [9]. This is evident, since tools needed for ensuring a high quality during the gathering of information can often not be separated from tools supporting a timely delivery during the transfer of information. Consequently, the second level only makes a distinction between the objective of achieving a high quality in $I E$ and of achieving cost efficiency of the IE activities. To achieve a high quality, the organization has to reduce existing variations in quality. For achieving cost efficiency, an adequate mean is to minimize the assets needed for IE activities and to reduce all kinds of existing waste. For establishing a sequence between the two goals a methodology from the area of 
production management is used. The so called Sand Cone Model shows that a company can only gain lasting capabilities by starting with a focus on quality. Cost efficiency is then a depended parameter of quality [10].

\subsection{3rd Level: Considering Organizational, Technical and Human Aspects}

The decomposition taking place on the third level considers the three major factors relevant for IE: organizational, technical, and human aspects [11]. Understanding their interdependencies is crucial for designing complex systems [11]. Organizational aspects refer to leadership principles and to the design of the structure and the workflows of an organization. The objective is to create organizational conditions supporting all IE activities [12]. Technical aspects include tools and methods necessary for supporting the organizational structure and its workflows. The objective is to provide a suitable infrastructure needed for IE [4]. Finally, issues closely related to the employees of the organization are summarized as human aspects [13]. This objective aims to assure the effectiveness of the employees. For establishing a sequence of the three goals, the numerous interdependencies between the aspects have to be taken into consideration. A possible example of a sequence is illustrated in a concept focusing on the development of knowledge management systems [14]. This concept identifies four pillars in the following order: leadership, organization, technology, and training. Since leadership is defined as a part of the organizational aspects, they will be the first in the sequence followed by technical factors. This is also in line with different findings in literature stating the role of technical aspects like ICT as a support to processes and larger systems (e.g.[15]). According to the mentioned concept [14], training follows the technical aspects. However, a practical validation shows that depending on the qualification of the employees, certain structures can be realized and techniques can be used. Conversely, the development and training of employees is influenced by the organizational structure and its workflows and by the used techniques. Consequently, human aspects would have to be mentioned at the first and last position. But since the selection and training of employees is usually not requiring or inflicting a change in the organization, the placement of human aspects is suitable to the most right.

\subsection{4th and 5th Level: Operationalization of the Different Aspects}

On the 4th and 5th level, an operationalization of the different organizational, technical and human aspects takes place. This provides a guideline for implementing or controlling measures and best practices. Figure 1 only shows the operationalization of some organizational aspects.

\section{Operationalization of organizational aspects}

An analysis of relevant organizational goals shows organizational culture and organizational structure as two critical success factors for all IE activities [16]. Establishing an organizational culture to support the IE is regarded as the most elementary factor to be improved in any organization $[16,12]$. It is therefore set as the first goal. A further decomposition on the $5^{\text {th }}$ level splits this goal into two 
elements to be achieved. The first is the integration of IM objectives into the strategy of the organization. This includes the communication of the importance of IE to the success of the company [17]. The second refers to the creation of common values in the whole organization by establishing norms for cooperation. This aims at building trust necessary for IE by establishing transparency, and by supporting an integrative collaboration throughout the organization [18].

To provide a suitable organizational structure supporting the IE is the second goal on the $4^{\text {th }}$ level. Designing the organizational structure requires to consider the existing organizational culture [12]. A decomposition of this goal on the $5^{\text {th }}$ level shows two major objectives: a formal and an informal integration of IE activities. A formal integration can be achieved by integrating IE tasks into the core and support processes of the company [16]. An informal integration aims at providing opportunities for an informal exchange of information between peoples in the organization [19]. Although no direct sequence between the two tasks can be defined, an excess of formalization might result in rigid processes reducing opportunities for informal IE [20]. Therefore, the formal integration is named first. It includes an analysis of existing processes, the identification of information sources and needs, the definition of responsibility, and the integration into daily work routines. Setting up informal relationships requires reducing contact barriers by creating possibilities for interactions. For instance, work rotation or temporary employee exchanges are suitable measures for communicating the needs and conditions of different partners.

\section{Operationalization of the technical aspects}

Technical aspects have to provide tools or infrastructure necessary for the IE activities. The most typical example is the provision of an ICT. From a quality perspective, such an ICT has to provide a high perceived functional usefulness, a high perceived reliability and a high perceived ease of use. These three aspects can be defined as goals for the $5^{\text {th }}$ level. A high functionality is the most fundamental aspect essential for the effectiveness of the ICT [21]. It has to support the user to exchange the right information at right process step and in the right format. In addition, it has to minimize the efforts of the user by matching relevant IE tasks with his workflow. This especially includes a reduction of idle times. One way to achieve a fast and process oriented IE is the use of a suitable ICT architecture [22]. A high perceived reliability can be realized by fulfilling the requirements for accessibility at different locations and times - keeping different time zones in mind. This includes both, guaranteeing availability of the ICT and providing all necessary access authorizations. For achieving high perceived ease of use the ICT has to be adjusted to the characteristics of the supported processes [23]. Finally, easy user interfaces have to be provided considering ergonomic norms like established in DIN EN ISO $9241[24]$.

\section{Operationalization of the human aspects}

Human aspects include two major goals: assuring the capabilities and the commitment of the employees [13]. Ensuring the capabilities is done by hiring suitable employees, by providing training and by reducing employee turnover [18]. In addition, external support can compensate a lack of certain capabilities, e.g. for 
structuring, articulating, searching, and using information [25]. Four types of competences can be distinguished and are arranged on the $5^{\text {th }}$ level: the provision of professional, organizational, technical and methodical competences.

For assuring the commitment of the employees, it is relevant to consider intrinsic and extrinsic types of motivation [26]. While intrinsic motivation is a result of all measures mentioned in this paper (operational, technical and human), extrinsic motivation can be achieved by selecting, combining and balancing suitable forms of compensation. However, offering extrinsic motivation can have a negative effect on the intrinsic motivation of the employees (crowding-out effect [26]). It is therefore crucial to identify those types of IE activities, which can be supported by extrinsic motivation without showing "side effects". On the $5^{\text {th }}$ level, three different forms of compensation can be distinguished: social, organizational and financial compensation. They can be used to motivate individual employees or whole organizational units (like subsidiaries).

\subsection{6th level: Application of the Goals and Measures to the IE Process}

On the $6^{\text {th }}$ level, all goals are applied to the different steps of the IE process: identification, gathering, transfer, search, and use of information. These steps are related to the lifecycle view of information [1]. A comprehensive description of this level can not be discussed within the context of this paper since it contains more than 40 possible goals and measures. An operationalization at the level of specific IE processes also allows for defining performance indicators which can easily be collected and used for benchmarking and for identifying existing weaknesses. In addition, it shows the impact of different best practices on the IE processes.

\section{Business Benefits and Conclusion}

The presented IMFC and its underlying methodology support companies in identifying goals and measures for improving IE. The IFC differs from other knowledge- and information management methodologies by connecting strategic, operational and application levels of IE and by showing the dependencies between the different goals and measures of those levels.

A discussion of the approach with the participating companies has shown that the application of this approach is quite intuitive and can support industrial service organizations in designing their IM activities. Firstly, it helps to identify the major goals of IE. After analyzing the current status of the IE activities, the approach supports the prioritization of goals and the balancing of appropriate measures. The goals can be made operational in a systematic way. The hierarchical structure allows to align all operational goals with the top-level objectives and the business strategy of the companies. As a final result, a set of mostly organizational and technical measures van be identified. However, discussions have shown that more specific information is needed about best practices and certain measures. Especially the extend, to which certain measures have been applied in different companies was of a special interest. Therefore, further examinations in this area are necessary. A survey 
conducted with different global organizations could help to find answers to some of those questions.

\section{References}

1. J. Schwarze, Informationsmanagement (NWB, Berlin, 1998).

2. F. Caldwell, The new enterprise knowledge management, VINE: The J. Information and Knowledge Management Systems. 36(2), 182-185 (2006).

3. P. Schuelke, Informationsmanagement im Service zur Verbesserung der Kommunikation mit den Unternehmensbereichen (FBK, Kaiserslautern, 2001).

4. H. Krcmar, Informationsmanagement (Springer, Berlin, 2005).

5. D.J. Greenwood, M. Levin, Action Research (Sage, Thousand Oaks, 1998).

6. N.P. Suh, Axiomatic Design (Oxford Uneversity Press, New York, 2001).

7. M.E. Porter, What Is Strategy?, Harvard Business Review, 74(6), (1996).

8. P. Schoensleben, Integral Logistics Management (Auerbach Publications, Boca Raton, 2007).

9. M. Eppler, Managing Information Quality (Springer, Berlin, 2006).

10. K. Ferdows, A. De Meyer, Lasting improvements in the manufacturing performance, J. Operations Management, 9(2), 168-184 (1990).

11.E. Ulich, Arbeitspsychologie (vdf, Zurich, 1994).

12.S.L. Pan, H. Scarbrough, A Socio-Technical View of Knowledge-Sharing at

Buckman Laboratories, J. Knowledge Management; 2(1), 55 - 66 (1998).

13.L. Argote, B. McEvily, R. Reagans, Managing knowledge in organizations, Management Science, 49(4), 571-582 (2003).

14.C. Baldanza, M. Stankosky, Knowledge management: an evolutionary architecture toward enterprise engineering, Proceedings of INCOSE (1999).

15.M. Mohamed, M. Stankosky, Knowledge management and information technology, $J$. Knowledge Management, 10(3), 103-116 (2006).

16. T. H. Davenport, L. Prusak, Das Praxisbuch zum Wissensmanagement (moderne industrie, Landsberg / Lech, 1998).

17.V. Decoene, W. Bruggeman, Strategic alignment and middle-level managers' motivation in a balanced scorecard setting, Int. J. Operations \& Production Management, 26(4), 429-448 (2006).

18. H. A. Artail, Application of KM measures to the impact of a specialized groupware system on corporate productivity and operations, Information \& Management, 43(4), 551-564 (2006).

19.P. Garg, R. Rastogi, New model of job design: motivating employees' performance, Journal of Management Development, 25(6), 572-587 (2005).

20.M. Hansen, The search-transfer problem, Admin. Sci. Quart., 44(1), 82-111 (1999).

21.G.-W. Bock, A. Kankanhalli, S. Sharma, Are norms enough?, European J. Information Systems, 15(4), 357 - 367 (2006).

22.M. Kirchmer, A.-W. Scheer, Business Process Automation, edited by A.-W. Scheer, F. Abolhassan, W. Jost, M. Kirchmer (Springer, Berlin, 2004).

23.H. Carter, Information Architecture, Work Study, 48(5), 182-185 (1999).

24.L. Bräutigam, W. Schneider, Projektleitfaden Software-Ergonomie (TSH, Wiesbaden, 2003).

25.J. Nadler, L. Thompson, L. Van Boven, Learning negotiation skills, Management Science, 49(4), 529-540 (2003).

26. E. L. Deci, Intrinsic Motivation (Plenum Press, New York, 1975). 